\title{
The Study of Test Method in Authentic Assessment of English Reading Ability
}

\author{
Lu Shan
}

Foreign Language College, Guangxi University of Science and Technology, Liuzhou, Guangxi, China (lzlushan@ 163.com)

\begin{abstract}
According to the real environment in daily life, the introduction of authentic assessment is an effective way to improve college students' English reading ability. There is a big difference between the test method in authentic assessment and that in traditional development of English reading ability. Firstly, according to the application of English for communication in daily life, the requirements for test in authentic assessment are discussed. Then, the application of some common test methods of authentic assessment in cultivating college students' English reading ability is discussed. Finally, some problems worthy of attention in choosing test method in authentic assessment of college students' English reading ability are put forward. This will provide basis for the introduction of authentic assessment in cultivating college students' English reading ability, which helps to improve the quality of teaching.
\end{abstract}

Keywords_-English reading ability, authentic assessment, test method

\section{英语阅读能力真实性评价中测试方法的研究}

\author{
卢珊 \\ 广西科技大学外国语学院, 柳州, 广西, 中国
}

摘＼cjkstart要＼cjkstart结合日常生活中的真实环境，引入真实性评价是提高大学生英语阅读能力的有效途径。真实性评价中的测试方法与传统 英语阅读能力培养中的测试方法存在差异。首先, 结合日常生活中应用英语进行交流交际的要求, 讨论了真实性评价中对于测试的要 求。接着, 讨论了真实性评价中几种常见测试方法在大学生英语阅读能力培养中的应用。最后, 提出了在大学生英语阅读能力真实性 评价中选择测试方法值得注意的几个问题。这将为在大学生英语阅读能力培养中引入真实性评价提供基础, 进而有助于相关教学环节 教学质量的提高。

关键词 英语阅读能力, 真实性评价, 测试方法

1. 引言

测试是教学中重要的反馈环节, 而在大学生英语能力 的培养中测试一直是重要的反馈渠道和评价方式, 因此针 对大学生的英语测试一直是关注的热点 ${ }^{[1]}$ 。特别是高考、 CET、研究生入学考试中英语科目成绩的重要性更增加了 对英语测试的关注。在相当长的一个时期中, 由于考试题 型中阅读理解比例相对较高, 这使得对英语阅读能力测试 的关注更为突出。

实际上英语能力的培养是为了学生日后工作学习能够 使用英语进行交际。因此, 在教学改革中, 从大学生英语

2013 年度广西高等教育教学改革工程项目支持（资助号: 2013JGB202)
阅读能力培养的角度出发, 阅读测试的真实性是非常重要 的内容 ${ }^{[2][3]}$ 。所以, 引入真实性评价, 强调大学生英语阅读 能力培养的过程与日后工作生活真实环境相一致, 这逐渐 成为一个发展方向。显然, 与传统的英语阅读测试相比, 引入真实性评价后, 必须在测试方法上进行改革才能保持 与真实情况的一致。

\section{2. 真实性评价中英语阅读测试的要求}

在传统的英语阅读测试中, 主要采取学生阅读一段文 字, 然后回答几个相关问题的形式。随着高考、CET、研 究生入学考试等标准化测试影响的不断扩大, 在上世纪末 本世纪初叶逐渐形成了一种较为固定的大学生英语阅读能 力测试方法。主要形式就是给出一段短文, 在学生阅读短 
文后给出几个问题, 包括词义解释、句子判断、短文意思 理解等多种内容, 给出 4 个选项构成选择题, 由学生选择 最为合理的答案。随着大学英语教学改革的深入, 现在逐 渐增加了回答问题等主观题的形式, 短文内容也进行了一 定的改革。但是, 从真实性评价的角度出发可以发现这样 的形式是无法满足真实性评价的要求的。

所谓真实性评价就是尽量模拟真实环境, 让学生在真 实使用英语的语言环境中锻炼、练习英语阅读能力。对应 的测试也是在真实环境中发生的。如果测试采用选择题的 形式, 那么在真实环境中就应该有这样的形式。按传统的 英语阅读测试方法, 在一些短文后会出现词义理解的选择 题, 这在现实生活中是非常罕见的现象。试想学生毕业工 作后, 看完一段英文材料, 指着其中一个单词问他是什么 意思, 这样的场景发生的几率极低。

所以, 从真实性评价的角度出发, 阅读能力的测试就 是真实环境中会出现的情况。测试的内容、测试的形式都 应该是真实环境中可能发生的情况。比如在地铁站购票的 测试中, 对学生的测试内容就应该是如何购票、如何选择 乘车方向, 如何选择进站、安检通道等实际问题, 测试的 形式就是让学生选择道路、自主购票等真实环境可能发生 的行为。

\section{3. 大学生英语阅读能力培养真实性评价中测试方法 的分析}

在真实环境中, 阅读的目的在于获取信息, 是为了进 行交流和决策做准备, 所以, 在引入真实性评价后测试的 方法可以表现为多种形式。结合日常生活的实际情况, 较 为常见的有以下几种。

\section{1 做出判断指导行动}

通过英语文本的阅读, 学生可以获得一定的信息, 依 据这些信息可以对一些问题进行判断, 进而针对特定的问 题形成决策。这也是传统英语阅读测试常用的测试方法, 要求学生根据阅读获取的信息进行判断。

值得注意的是, 在真实性评价中测试方法与传统测试 的不同之处主要在于测试方法中测试目的的重要性不同。 传统英语阅读测试中, 除了测试学生对英文信息的应用外, 往往还将测试的内容扩展到对文字信息的理解, 对英语段 落的分析, 语法的分析, 甚至词汇的理解。而在真实性评 价中, 这类测试方法更突出最后对阅读信息的应用。如在 对产品说明书的阅读中, 传统测试可能还包括对产品功效 的理解、对产品介绍中句子的分析等内容; 而在真实性评 价中, 一般只要求学生根据产品说明书做出判断该产品是
否适用于某些特定的场合。显然, 真实性评价更接近学生 今后工作中英语阅读所涉及的真实情况。

\section{2 参与讨论阐述自己观点}

在现实生活中, 在阅读之后往往是与别人进行交流。 在交流的过程中, 需要根据自己阅读获取的信息阐述自己 的理解, 并结合自己已有的知识经验阐述自己的观点与别 人进行讨论。所以在真实性评价中, 可以采取类似表现型 评价的方式, 让学生自己阐述自己的观点, 展现自己阅读 所获得的信息。

在这类评价方式中，主要是模拟阅读后交流的过程。 那么评价的内容就是能否充分的与别人进行交流, 评价的 标准则是结合阅读材料充分展示自己的观点。所以, 在这 样的评价方式中, 学生参与讨论的程度与对信息理解的正 确性全面性一样重要。

\section{3 询问别人进一步获取信息}

在真实环境中, 人常常处于一个多媒体的环境, 文字、 声音等各种信息整合在同一空间时间中。即使是文字信息 也不可能如传统阅读理解那样整合在一篇文章中, 而是散 落于各个物理空间。如在大型技术展会中, 大量的文字信 息分布在各个空间, 这时候阅读的目的是了寻找自己感兴 趣的信息进一步询问。因此, 可以采取让学生询问的方式 进行测试。

在这类测试方式中, 首先给学生确定一定的任务; 然 后模拟真实环境给出一定的英文材料, 这主要通过视频、 文字给出; 最后由教师扮演咨询人员, 由学生根据所获得 的材料进行咨询。为减少学生英语口语表达的差异, 可以 考虑允许学生用母语进行提问。根据学生的提问了解学生 在真实环境中的阅读能力。

\section{4. 测试方法选择中值得注意的问题}

在教学实践中, 每种测试方法都有多种的测试形式, 即使采用同样的测试形式在具体课堂活动、测试活动的组 织上也可以因为细节存在差异。但是, 在测试方法的具体 选择、细节的组织中以下几点是值得注意的。

\section{1 测试方法的可行性}

测试最后必须给出一个测试的结果, 因此测试最后是 需要进行评价的。在真实性评价中许多测试活动都是建立 在学生有一定的理解基础上, 因此, 测试活动的选择要考 虑学生的基础, 要具有一定的可行性。 


\section{2 测试评价标准的客观性}

测试的一个重要目的在于给予反馈, 这是提高教学质 量的关键, 在引入真实性评价后测试评价的客观性受到极 大挑战。事实上, 在现实生活中对于阅读效果的评价一般 很少发生, 对于一个人英语阅读能力的评价一般是基于一 系列活动后得出的主观印象。但是, 这是与测试的目的相 违背的, 所以在测试标准的设置中除了考虑真实性还需要 考虑对学生能力的客观反映。

\section{5. 小结}

本文讨论了大学生英语阅读能力的真实性测试, 这是
在大学英语阅读中进行真实性评价的基础, 将有助于提高 大学生阅读能力的培养质量。

\section{参考文献(References)}

[1] Luo Qianli. Literature review of the assessment system research in foreign language teaching. Journal of Sichuan college of education, vol.25, 10 pp. 104-106, 2009.

[2] Zhao Jing. Analysis on veracity of reading comprehension in college entrance examination. Journal of Guizhou normal college (social science), vol.20, 2 pp. 72-75, 2010

[3] Zhu Xiaohua. A study of authenticity of a reading achievement test. Journal of Weinan normal university. vol. 29, pp. 62-65, 2014. 